\title{
The bipartite graphs of abelian dessins d'enfants
}

\author{
Rubén A. Hidalgo * \\ Departamento de Matemática, Universidad Técnica Federico Santa María \\ Valparaíso, Chile
}

Received 25 April 2012, accepted 31 July 2012, published online 21 November 2012

\begin{abstract}
Let $S$ be a closed Riemann surface and let $\beta: S \rightarrow \widehat{\mathbb{C}}$ be a regular branched holomorphic covering, with an abelian group as deck group, whose branch values are contained in the set $\{\infty, 0,1\}$. Three dessins d'enfants are provided by $\beta^{-1}([0,1]), \beta^{-1}([1, \infty])$ and $\beta^{-1}([0, \infty])$. In this paper we provide a description of the bipartite graphs associated to these dessins d'enfants using simple arguments.
\end{abstract}

Keywords: Dessins d'enfants, Belyi curves, Algebraic curves, Riemann Surfaces.

Math. Subj. Class.: 11G32, 14H37, 30F10

\section{Introduction}

As a consequence of the Riemann-Roch theorem, there is a bijective correspondence between isomorphism classes of closed Riemann surfaces and isomorphism classes of complex algebraic curves. A closed Riemann surface $S$ is called a Belyi curve if there is a non-constant meromorphic function $\beta: S \rightarrow \widehat{\mathbb{C}}$ whose branch values are contained in $\{\infty, 0,1\}$. The function $\beta$ is called a Belyi function for $S$ and $(S, \beta)$ is called a Belyi pair. If the branch orders of $\beta$ at 0,1 and $\infty$ are $p, q$ and $r$, respectively, then we say that the Belyi pair $(S, \beta)$ is of type $(p, q, r)$.

If $(S, \beta)$ is a Bely pair, then the pre-image $D_{1}=\beta^{-1}([0,1])\left(D_{2}=\beta^{-1}([1, \infty])\right.$ and $D_{3}=\beta^{-1}([0, \infty])$, respectively) defines a dessin d'enfant on $S$ (see [7]), that is, a bipartite map on $S$ (the pre-image of 0 are the white vertices and the pre-image of 1 are the black vertices of $D_{1}$ ). Conversely, by the Uniformization Theorem, each dessin d'enfant $D$ on a closed orientable surface induces a unique (up to isomorphisms) Riemann surface structure $S$ on it and a Belyi map $\beta: S \rightarrow \widehat{\mathbb{C}}$ so that $D$ and $\beta^{-1}([0,1])$ are equivalent bipartite maps on $S$. A famous result due to Belyi [1,2] states that a closed Riemann surface $S$ is a Belyi curve if and only if $S$ can be defined by an algebraic curve over $\overline{\mathbb{Q}}$. This relationship was

\footnotetext{
*Partially supported by Projects Fondecyt 1110001 and UTFSM 12.11.01.

E-mail address: ruben.hidalgo@usm.cl (Rubén A. Hidalgo)
} 
observed by Grothendieck in his famous Esquisse d'un programme [3] to propose a study of the structure of the absolute Galois group $\mathrm{Gal}(\overline{\mathbb{Q}} / \mathbb{Q})$ by its action on the dessins d'enfants. As a consequence, a natural link between Galois theory, Belyi pairs and dessins d'enfants appears and, moreover, Galois invariants should be expressed in a purely combinatorial form. Unfortunately, the action of $\operatorname{Gal}(\overline{\mathbb{Q}} / \mathbb{Q})$ on dessins d'enfants is not well understood.

A particular class of dessins d'enfants are those produced by Belyi pairs $(S, \beta)$ for which $\beta$ is a regular branched holomorphic cover; in which case we say that $S$ is a quasiplatonic curve. Wolfart [8] noticed that quasiplatonic curves (and also the corresponding regular dessins d'enfants) are definable over their field of moduli. In the particular case when the deck group of $\beta$ is an abelian group, we say that $S$ is an abelian quasiplatonic curve, that $(S, \beta)$ is an abelian Belyi pair (the corresponding dessins d'enfants are called abelian dessins d'enfants). In this case, if $(p, q, r)$ is the type of $(S, \beta)$, then we say that signature $(0 ; p, q, r)$ is an abelian triangular signature. In [4] it was noticed that every abelian Belyi pair (and the corresponding abelian dessins d'enfants) can be defined over $\mathbb{Q}$, that is, they are fixed points for the action of $\mathrm{Gal}(\overline{\mathbb{Q}} / \mathbb{Q})$. In this paper we describe, using simple arguments, the underlying bipartite graphs of the abelian dessins d'enfants (see Theorem 1). Next, we proceed to describe a couple of classical examples.

If $n, m, d$ are positive integers, then we denote by $K_{n, m}^{d}$ the bipartite graph obtained from the complete bipartite graph $K_{n, m}$ by replacing each edge by $d$ edges. In this way, $K_{n, m}^{1}=K_{n, m}$.

(1) If $(S, \beta)$ is an abelian Belyi pair of type $(k, k, k)$, where $k \geq 2$ is an integer, and whose deck group of $\beta$ is $\mathbb{Z}_{k}^{2}$, then $(S, \beta)$ is isomorphic to $\left(F_{k}, \beta_{k}\right)$, where $F_{k}$ is the classical Fermat curve $\left\{x^{k}+y^{k}+z^{k}=0\right\} \subset \mathbb{P}^{2}, \beta_{k}([x: y: z])=-(y / x)^{k}$ and the deck group of $\beta_{k}$ is the abelian group generated by $a([x: y: z])=\left[\omega_{k} x: y: z\right]$ and $b([x: y: z])=\left[x: \omega_{k} y: z\right]$, where $\omega_{k}=e^{2 \pi i / k}$. The fixed points of $a$ (respectively, $b$ and $a b$ ) are given by the $k$ points in $\beta_{k}^{-1}(\infty)$ (respectively, $\beta_{k}^{-1}(0)$ and $\beta_{k}^{-1}(1)$ ). The abelian dessins d'enfants $D_{1}=\beta_{k}^{-1}([0,1])$, $D_{2}=\beta_{k}^{-1}([1, \infty])$ and $D_{3}=\beta_{k}^{-1}([0, \infty])$ have as bipartite graph the complete bipartite graph $K_{k, k}^{1}[5,6]$.

(2) If $(\widehat{\mathbb{C}}, \beta)$ is an abelian Belyi pair, with deck group $H$, then either $H \cong \mathbb{Z}_{n}$ or $H \cong \mathbb{Z}_{2}^{2}$. The associated bipartite graphs of the corresponding abelian dessins d'enfants are in the first case equal to $K_{1, n}^{1}$ and $K_{1,1}^{n}$ and in the second case equal to $K_{2,2}^{1}$.

Theorem 1 below generalizes the above to the case of abelian dessins d'enfants of any type.

Theorem 1. Let $(S, \beta)$ be an abelian Belyi pair of type $(p, q, r)$ and let $d$ be the degree of $\beta$. Then the bipartite graphs associated to the three abelian dessins d'enfants are given by

$$
\mathcal{G}_{1}=K_{d / p, d / q}^{p q / d}, \quad \mathcal{G}_{2}=K_{d / q, d / r}^{q r / d}, \quad \mathcal{G}_{3}=K_{d / p, d / r}^{p r / d}
$$

Remark 2. Particular classes of abelian dessins d'enfants are those provided by the maximal ones with respect to its type (as the case provided by classical Fermat curves). An abelian dessin d'enfant associated to an abelian Belyi pair $(S, \beta)$, with abelian group $H$ as deck group of $\beta$, is called an homology dessin d'enfant (and $(S, \beta)$ is called an homology Belyi pair) if there is no an abelian Belyi pair $(R, \eta)$, with abelian group $G$ as deck group of $\eta$, so that $S=R / L$ for some non-trivial subgroup $L<G$ acting freely on $R$ with $H=G / L$. If $(S, \beta)$ is an homology Belyi pair, then equations over $\mathbb{Q}$ for $S$ and $\beta$ were found in [4]. 
Clearly every abelian dessin d'enfant is covered by an homology dessin d'enfant of the same type. If the genus of $S$ is at least two, then a homology Belyi pair $(S, \beta)$ of type $(p, q, r)$ can be uniformized as follows. Let $\Gamma$ be a Fuchsian group of signature $(0 ; p, q, r)$ and let $\Gamma^{\prime}$ be its derivative subgroup. Then $(S, \beta)$ is equivalent to $\left(\mathbb{H}^{2} / \Gamma^{\prime}, \beta_{\Gamma}\right)$, where $\beta_{\Gamma}$ is the natural quotient map $\mathbb{H}^{2} / \Gamma^{\prime} \rightarrow \mathbb{H}^{2} / \Gamma$. In this way, not only the bipartite graphs may be described, but also the corresponding dessins d'enfants.

\section{Proof of Theorem 1}

Let $(S, \beta)$ be an abelian Belyi pair of type $(p, q, r)$ and let $H$ be the abelian group being the deck group of $\beta$ (so $d=|H|$, the order of $H$ ).

Let $D_{1}$ be the dessin d'enfant whose edges are the pre-images under $\beta$ of the arc $[0,1]$, the black vertices are the pre-images of 0 and the white vertices are the pre-images of 1 . The number of black vertices is equal to $|H| / p$, the number of white vertices is equal to $|H| / q$ and the number of faces is $|H| / r$. The degree of a black vertex is $p$, the degree of a white vertex is $q$ and the degree of a face is $r$.

Let $x_{1} \in S$ (respectively, $y_{1} \in S$ ) be such that $\beta\left(x_{1}\right)=0$ (respectively, $\beta\left(y_{1}\right)=1$ ). Let $\mathbb{Z}_{p} \cong\langle a\rangle<H$ (respectively, $\mathbb{Z}_{q} \cong\langle b\rangle<H$ ) be the $H$-stabilizer of $x_{1}$ (respectively, the $H$-stabilizer of $\left.y_{1}\right)$. As $H$ acts transitively on $\beta^{-1}(0)$ (respectively, $\beta^{-1}(1)$ ) and $H$ is abelian, we may see that:

1. the $H$-stabilizer of every point in $\beta^{-1}(0)$ (respectively, $\left.\beta^{-1}(1)\right)$ is $\langle a\rangle$ (respectively, $\langle b\rangle)$;

2. $H=\langle a, b\rangle$;

3. $\langle b\rangle$ (respectively, $\langle a\rangle)$ acts transitively on $\beta^{-1}(0)$ (respectively, $\beta^{-1}(1)$ ).

As there is a black vertex and a white vertex connected with an edge, condition (3) above ensures that every black vertex and every white vertex is connected by an edge.

Again from (3), the $\langle b\rangle$-stabilizer of $x_{1}$ (respectively, the $\langle a\rangle$-stabilizer of $y_{1}$ ) is its cyclic subgroup of $\langle b\rangle$ (respectively, $\langle a\rangle$ ) of order $p q /|H|$. It follows that every pair of black and white vertices are connected with $p q /|H|$ edges.

All the above information permits to obtain that the graph associated to $D_{1}$ is the bipartite graph

$$
\mathcal{G}_{1}=K_{|H| / p,|H| / q}^{p q /|H|}
$$

Similarly, let $D_{2}$ (respectively, $D_{3}$ ) be the dessin d'enfant obtained as the pre-image of the arc $[1, \infty]$ (respectively, $[\infty, 0]$ ) and the corresponding graph $\mathcal{G}_{2}$ (respectively, $\mathcal{G}_{3}$ ). Then, working in the same way as for $D_{1}$ one obtains that

$$
\mathcal{G}_{2}=K_{|H| / q,|H| / r}^{q r / H \mid}, \quad \mathcal{G}_{3}=K_{|H| / p,|H| / r}^{p r / H \mid} .
$$

Remark 3. It is well know that a signature $(0 ; p, q, r)$ is an abelian triangular signature if and only if $\operatorname{lcm}(p, q, r)=\operatorname{lcm}(p, q)=\operatorname{lcm}(p, r)=\operatorname{lcm}(q, r)$, where $1 \mathrm{~cm}$ stands for the least common multiple. In that case we may write

$$
p=A A_{12} A_{13}, q=A A_{12} A_{23}, r=A A_{13} A_{23},
$$

where $\operatorname{gcd}\left(A_{12}, A_{13}\right)=\operatorname{gcd}\left(A_{12}, A_{23}\right)=\operatorname{gcd}\left(A_{13}, A_{23}\right)=1$ and $A=\operatorname{gcd}(p, q, r)$, where $\operatorname{gcd}$ stands for the greatest common divisor. 
In [4] we proved that if $(S, \beta)$ is an homology Belyi pair, then $H \cong \mathbb{Z}_{A} \times \mathbb{Z}_{\mu}$, where $\mu=\operatorname{lcm}(p, q, r)=A A_{12} A_{13} A_{23}$. In particular, the bipartite graphs in Theorem 1 are given by

$$
\mathcal{G}_{1}=K_{A A_{23}, A A_{13}}^{A_{12}}, \quad \mathcal{G}_{2}=K_{A A_{13}, A A_{12}}^{A_{23}}, \quad \mathcal{G}_{3}=K_{A A_{23}, A A_{12}}^{A_{13}} .
$$

In the general situation, that is, for any abelian Bely pair of type $(p, q, r)$ with $\beta$ of degree $d$, the bipartite graphs of the three dessins d'enfants are given by

$$
\mathcal{G}_{1}=K_{A A_{23} / l, A A_{13} / l}^{l A_{12}}, \quad \mathcal{G}_{2}=K_{A A_{13 / l}, A A_{12} / l}^{l A_{23}}, \quad \mathcal{G}_{3}=K_{A A_{23} / l, A A_{12} / l}^{l A_{13}},
$$

where $l=A^{2} A_{12} A_{13} A_{23} / d$.

\section{Acknowledgments}

The author would like to thank the referee for his/her careful reading and the suggested corrections to the first version.

\section{References}

[1] G. V. Belyi, Galois extensions of a maximal cyclotomic field, Izv. Akad. Nauk SSSR Ser. Mat. 43 (1979), 267-276.

[2] G. V. Belyi, A new proof of the three-point theorem, Mat. Sb. 193 (2002), 21-24.

[3] A. Grothendieck, Esquisse d'un programme, in: L. Schneps and P. Lochak (eds.), Geometric Galois actions 1. The Inverse Galois Problem, Moduli Spaces and Mapping Class Groups, London Math. Soc. Lecture Note Ser. 242, pages 5-48, with an English translation on pp. 243-283, Cambridge Univ. Press, Cambridge, 1997.

[4] R.A. Hidalgo, Homology closed Riemann surfaces, Quarterly Journal of Math. (2011), doi: 10.1093/qmath/har026

[5] G. A. Jones and M. Streit, Galois groups, monodromy groups and cartographic groups, in: L. Schneps and P. Lochak (eds.), Geometric Galois Actions 2. The Inverse Galois Problem, Moduli Spaces and Mapping Class Groups. London Math. Soc. Lecture Note Ser. 243, pages 25-65. Cambridge Univ. Press, Cambridge, 1997.

[6] G. Ringel and J. W. T. Youngs, Das Geschlecht des vollständigen dreifärbbaren Graphen, Comm. Math. Helv. 45 (1970), 152-158.

[7] D. Singerman and J. Wolfart, Cayley Graphs, Cori Hypermaps, and Dessins d'Enfants, Ars Math. Contemp. 1 (2008), 144-153.

[8] J. Wolfart, $\mathrm{ABC}$ for polynomials, dessins d'enfants and uniformization-a survey, in: Elementare und analytische Zahlentheorie, Schr. Wiss. Ges. Johann Wolfgang Goethe Univ. Frankfurt am Main, 20, Franz Steiner Verlag Stuttgart, Stuttgart, 2006, pp. 313-345. 